\title{
Exact Solutions of Nonlinear Partial Differential Equations with $\exp (-\varphi(\zeta))-$ Expansion Method
}

\author{
Filiz Taşcan, Arzu Akbulut \\ Eskişehir Osmangazi Universitesi, Fen- Edebiyat Fakültesi, Matematik-Bilgisayar Bölümü, \\ Eskişehir-TÜRKIYYE \\ e-posta : ftascan@ogu.edu.tr, ayakut1987@hotmail.com
}

Geliş Tarihi: 18.04.2016 ; Kabul Tarihi: 24.01.2017

\begin{tabular}{cl} 
Keywords & Abstract \\
\cline { 2 - 3 } $\begin{array}{c}\text { Exact solutions; } \\
\text { Symbolic }\end{array}$ & $\begin{array}{l}\text { The aim of this paper is obtaining the exact solutions of the Bogoyavlenskii equation and the modified } \\
\text { KdV-Zakharov-Kuznetsev equation with the help of the exp }(-\phi(\zeta)) \text {-expansion method. Solutions are } \\
\text { computation; Partial } \\
\text { differential equation }\end{array}$ \\
$\begin{array}{l}\text { obtained with different forms of functions as hyperbolic, trigonometric and rational functions. } \\
\text { Discussed mod is useful for obtaining solutions of nonlinear equations in mathematical physics and } \\
\text { engineering. }\end{array}$
\end{tabular}

\section{Lineer Olmayan Kısmi Diferensiyel Denklemlerin exp(- $\phi(\zeta))-$ Açılım Metodu ile Tam Çözümleri}

\author{
Anahtar kelimeler Özet \\ Tam çözümler; \\ Sembolik hesaplama; \\ Kısmi diferensiyel \\ denklemler.

\begin{abstract}
Bu çalışmada Bogoyavlenskii denklemi ve modifiye edilmiş KdV-Zakharov-Kuznetsev denkleminin tam çözümleri exp(- $\phi(\zeta))$-açılım metodu yardımıyla bulunmuştur. Bulunan çözümler birbirinden farklı fonksiyonlardır ve hiperbolik, trigonometrik ve rasyonel fonksiyonlar şeklinde ifade edilmiştir. Ele alınan yöntem matematiksel fizik ve mühendislikte kullanılan lineer olmayan denklemlerin çözümü için çözümlerinin bulunması için oldukça kullanışlıdır.
\end{abstract}

(C) Afyon Kocatepe Üniversitesi

\section{Introduction}

Nonlinear partial differential equations (NLPDEs) with time $t$ as one of the independent variables have become useful for natural phenomena of science and engineering. NLPDEs are not arise only from many fields of mathematics, but also from different discipline such as fluid mechanics, plasma physics, optical fibers, solid state physics, chemical kinematic, chemical physics and geochemistry. Exact solutions of nonlinear partial differential equations play an important role in many various areas of natural science. Studies of exact solutions of nonlinear partial differential equations are becoming attractive in nonlinear science. Therefore, many effective and powerful methods established and improved, such as Jacobi elliptic function method (Liu and Fan, 2005),
Weierstrass elliptic function method (Achab, 2016), Darboux and Backlund transform (Matveev and Salle, 1991), symmetry reduction method (Taşcan and Yakut, 2015; Bluman and Kumei, 1989), the tanh method (Bekir and Çevikel, 2009), auxiliary method (Kaplan et al., 2015), extended tanh method (Wazwaz, 2008), sine-cosine method (Taşcan and Bekir, 2009), homogeneous balance method (Eslami et. al., 2014), variational iteration method (Ghaneai and Hosseini, 2016), expfunction method (Zayed and Abdelaziz, 2011), the $\left(G^{\prime} / G\right)$-expansion method (Khan and Akbar, 2014), the modified simple equation method (Kaplan et. Al., 2015), first integral method (Taghizadeh et. al., 2011), trigonometric function series method (Zhang, 2008), exp $(-\phi(\zeta))$ expansion method (Khan and Akbar, 2014; Khan and Akbar, 2013; Hafez et. al., 2014;Abdelrahman et. al., 2015). 
In this paper, we will search the applicability and effectivity of the $\exp (-\phi(\zeta))$ expansion method on nonlinear partial differential equations.

In literature, exp $(-\phi(\zeta))$ expansion method is used for obtaining exact solutions of partial differential equations. For example; modified Benjamin-Bona-Mahony equation by Khan and Akbar (Khan and Akbar, 2013), (1+1) dimensional Kaup-Kupershmidt equation by Roshid et. al. (Roshid et. al., 2015), generalized Hirota-Satsuma couple KdV system by Khater (Khater,2016), Nonlinear Dynamics of Microtubules- A New Model and The Kundu- Eckhaus Equations by Zahran and Khater (Zahran and Khater, 2015). All obtained solutions are hyperbolic, trigonometric and rational function solutions.

In section 2, we will define needed preliminary information about the $\exp (-\phi(\zeta))$ - expansion method. Next section, we will utilize this method to the Bogoyavlenskii equation and the modified KdV-Zakharov-Kuznetsev equation. For this application firstly we will reduce these nonlinear partial differential equations to nonlinear ordinary differential equations with wave variable $\zeta=x+y-w t$. Then, exact solutions will be obtained in the forms of hyperbolic, trigonometric, rational functions for given equations. We have seen that our obtained solutions more wider than other obtained solutions in literature (Malik et.al,2010;Najafi et.al, 2012; Naher et.al. 2013).

Finally, some conclusions will be given.

\section{The $\exp (-\phi(\zeta))$ expansion method}

More and new exact wave solutions of NLPDEs can be obtain with the $\exp (-\phi(\zeta))$ expansion method. In this section we will give necessary information about $\exp (-\phi(\zeta))$ expansion method.

Think that a nonlinear partial differential equation in the following form

$$
P\left(u, u_{t}, u_{x}, u_{y}, u_{t t}, u_{x t}, u_{x x}, u_{t y}, u_{x y}, u_{y y} \ldots\right)=0
$$

where $x, y, t$ are independent variablaes and $u(x, y, t)$ is dependent variable. We can use the wave variable $\zeta=x+y-w t$ (here $w$ is constant) for reducing Eq. (1) to a nonlinear ordinary differential equation as

$$
Q\left(u, u^{\prime}, u^{\prime \prime}, \ldots\right)=0
$$

where the prime denotes the derivation with respect to $\zeta$.

We should take the solution of ODE (2) in the following form

$$
u(\zeta)=\sum_{i=0}^{n} \alpha_{i}(\exp (-\phi(\zeta)))^{i}
$$

here $a_{i} \quad(0 \leq i \leq n)$ are constants and they will be determined later, $\alpha_{n} \neq 0$, and the balance term $n$ can be calculated by using homogeneous balance method between the highest order derivatives and the nonlinear terms appearing in ODE (2). $\phi=\phi(\zeta)$ satisfies the following ODE

$$
\phi^{\prime}(\zeta)=\exp (-\phi(\zeta))+\mu \exp (\phi(\zeta))+\lambda
$$

where $\lambda, \mu$ are constants.

Eq. (4) gives the following solutions;

1) If we take $\lambda^{2}-4 \mu>0, \mu \neq 0$ in ODE (4) we obtain following hyperbolic function solutions.

$$
\phi_{1}(\zeta)=\ln \left(\frac{-\sqrt{\left(\lambda^{2}-4 \mu\right)} \tanh \left(\frac{\sqrt{\left(\lambda^{2}-4 \mu\right.}}{2}(\zeta+k)\right)-\lambda}{2 \mu}\right)
$$

and

$$
\phi_{2}(\zeta)=\ln \left(\frac{-\sqrt{\left(\lambda^{2}-4 \mu\right)} \operatorname{coth}\left(\frac{\sqrt{\left(\lambda^{2}-4 \mu\right.}}{2}(\zeta+k)\right)-\lambda}{2 \mu}\right) .
$$

2) If we use $\lambda^{2}-4 \mu<0, \mu \neq 0$ in ODE (4) we get following trigonometric function solutions.

$$
\phi_{3}(\zeta)=\ln \left(\frac{\sqrt{\left(4 \mu-\lambda^{2}\right)} \tan \left(\frac{\sqrt{\left(4 \mu-\lambda^{2}\right)}}{2}(\zeta+k)\right)-\lambda}{2 \mu}\right)
$$

and

$$
\phi_{4}(\zeta)=\ln \left(\frac{\sqrt{\left(4 \mu-\lambda^{2}\right)} \cot \left(\frac{\sqrt{\left(4 \mu-\lambda^{2}\right)}}{2}(\zeta+k)\right)-\lambda}{2 \mu}\right)
$$

3) When $\lambda^{2}-4 \mu>0, \mu=0, \lambda \neq 0$, rational function solution is given by 


$$
\phi_{5}(\zeta)=-\ln \left(\frac{\lambda}{\exp (\lambda(\zeta+k))-1}\right)
$$

4) When $\lambda^{2}-4 \mu=0, \mu \neq 0, \lambda \neq 0$, we get following solution

$$
\phi_{6}(\zeta)=\ln \left(-\frac{2(\lambda(\zeta+k)+2)}{\lambda^{2}(\zeta+k)}\right)
$$

5) When $\lambda^{2}-4 \mu=0, \mu=0, \lambda=0$, solution is obtained in the following form

$$
\phi_{7}(\zeta)=\ln (\zeta+k)
$$

where $k$ is arbitrary constant and we will find values of $k$ later.

If we substitute Eq. (3) into Eq. (2), we have polynomial of function $\exp (-\phi(\zeta))$. If we equate the coefficients of all same powers of $\exp (-\phi(\zeta))$ to zero, we get a set of algebraic equations. Then we can find values of $\alpha_{i}(0 \leq i \leq n), \quad \lambda, \quad \mu, \quad w$ with the help of obtained a set of algebraic equations. Substituting these founded values into Eq. (3) along with solutions of Eq. (4), we find exact solutions of Eq. (1) (Hafez and Akbar, 2015).

\section{Applications}

\subsection{The Bogoyavlenskii equation}

In this subsection we will deal with the Bogoyavlenskii equation. The Bogoyavlenskii equation is modified version of breaking soliton equation given by

$$
4 u_{x t}+8 u_{x} u_{x y}+4 u_{y} u_{x x}+u_{x x x y}=0 .
$$

describes the $(2+1)$-dimensional interaction of a Riemann wave propagating along the $y$-axis with a long wave along the x-axis (Malik et. al., 2012;Peng and Shen, 2006). Bogoyavlenskii equation is given in the following form

$$
\begin{array}{r}
4 u_{t}+u_{x x y}-4 u^{2} u_{y}-4 u_{x} v=0 \\
u u_{y}=v_{x} .
\end{array}
$$

Now we will obtain Bogoyavlenskii equation's exact solutions with $\exp (-\phi(\zeta))$ expansion method. Using the following transformation

$$
u(x, y, t)=u(\zeta), \zeta=x+y-w t,
$$

we obtain

$$
\begin{aligned}
-4 w u^{\prime}+u^{\prime \prime \prime}-4 u^{2} u^{\prime}-4 u^{\prime} v & =0 \\
u u^{\prime} & =v^{\prime} .
\end{aligned}
$$

Integrating second ODE once with respect to $\zeta$, then putting founded value of in the first equation, the Sys. (14) reduces to following ODE

$$
-4 w u^{\prime}+u^{\prime \prime \prime}-6 u^{2} u^{\prime}=0
$$

and integrating founded ODE once with respect to $\zeta$ and equating constant of integration to zero, the Eq. (12) reduces to following ODE

$$
u^{\prime \prime}-2 u^{3}-4 w u=0
$$

where the prime denotes the derivation with respect to $\zeta$. Homogeneous balance is 1 in Eq. (16) and then

$$
u(\zeta)=\alpha_{0}+\alpha_{1} \exp (-\phi(\zeta))
$$

When we substitute Eq. (17) with the help of Eq. (4) into Eq. (16), then equating the coefficients of $\exp (-\phi(\zeta))^{j}$ to zero $(j \geq 0)$, we get following system

$$
\begin{aligned}
-4 w \alpha_{0}-2 \alpha_{0}^{3}+\alpha_{1} \mu \lambda & =0, \\
-6 \alpha_{0}^{2} \alpha_{1}+2 \alpha_{1} \mu-4 w \alpha_{1}+\alpha_{1} \lambda^{2} & =0, \\
-6 \alpha_{0} \alpha_{1}^{2}+3 \alpha_{1} \lambda & =0, \\
-2 \alpha_{1}^{3}+2 \alpha_{1} & =0 .
\end{aligned}
$$

From system (18), we get

$$
\begin{array}{rrr}
w=w, & \alpha_{0}=0, & \alpha_{1}=0, \\
w=-\frac{\alpha_{0}^{2}}{2}, & \alpha_{0}=\alpha_{0}, & \alpha_{1}=0, \\
w=-\frac{\lambda^{2}}{8}+\frac{\mu}{2}, & \alpha_{0}=\frac{\lambda}{2}, & \alpha_{1}=1, \\
w=-\frac{\lambda^{2}}{8}+\frac{\mu}{2}, & \alpha_{0}=-\frac{\lambda}{2}, & \alpha_{1}=-1 .
\end{array}
$$

We can find solution of Eq. (12) only using third and fourth cases in (19) because of $\alpha_{1} \neq 0$. When

$$
w=-\frac{\lambda^{2}}{8}+\frac{\mu}{2}, \alpha_{0}= \pm \frac{\lambda}{2}, \alpha_{1}= \pm 1,
$$

solution of Eq. (12) is given by

$$
u(\zeta)= \pm \frac{\lambda}{2} \pm 1 \exp (-\phi(\zeta))
$$

where 


$$
\zeta=x-\left(-\frac{\lambda^{2}}{8}+\frac{\mu}{2}\right) t .
$$

Substituting Eqs. (5-11) into Eq. (20), we obtain the following seven exactsolutions of Eq. (12).

1) When $\lambda^{2}-4 \mu>0, \mu \neq 0$;

$$
\begin{aligned}
& u_{1}(\zeta)= \pm \frac{\lambda}{2} \mp \frac{2 \mu}{\sqrt{\lambda^{2}-4 \mu} \tanh \left(\frac{1}{2} \sqrt{\lambda^{2}-4 \mu}(\zeta+k)\right)+\lambda}, \\
& v_{1}(\zeta)=\frac{1}{2}\left( \pm \frac{\lambda}{2} \mp \frac{2 \mu}{\sqrt{\lambda^{2}-4 \mu} \tanh \left(\frac{1}{2} \sqrt{\lambda^{2}-4 \mu}(\zeta+k)\right)+\lambda}\right)^{2}
\end{aligned}
$$

and

$$
\begin{aligned}
& u_{2}(\zeta)= \pm \frac{\lambda}{2} \mp \frac{2 \mu}{\sqrt{\lambda^{2}-4 \mu} \operatorname{coth}\left(\frac{1}{2} \sqrt{\lambda^{2}-4 \mu}(\zeta+k)\right)+\lambda}, \\
& v_{2}(\zeta)=\frac{1}{2}\left( \pm \frac{\lambda}{2} \mp \frac{2 \mu}{\sqrt{\lambda^{2}-4 \mu} \operatorname{coth}\left(\frac{1}{2} \sqrt{\lambda^{2}-4 \mu}(\zeta+k)\right)+\lambda}\right)^{2}
\end{aligned}
$$

where

$$
\zeta=x-\left(-\frac{\lambda^{2}}{8}+\frac{\mu}{2}\right) t .
$$

2) When $\lambda^{2}-4 \mu<0, \mu \neq 0$;

$$
\begin{gathered}
u_{3}(\zeta)= \pm \frac{\lambda}{2} \pm \frac{2 \mu}{\sqrt{4 \mu-\lambda^{2}} \tan \left(\frac{1}{2} \sqrt{4 \mu-\lambda^{2}}(\zeta+k)\right)-\lambda}, \\
v_{3}(\zeta)=\frac{1}{2}\left( \pm \frac{\lambda}{2} \pm \frac{2 \mu}{\sqrt{4 \mu-\lambda^{2}} \tan \left(\frac{1}{2} \sqrt{4 \mu-\lambda^{2}}(\zeta+k)\right)-\lambda}\right)^{2}
\end{gathered}
$$

and

$$
\begin{aligned}
& u_{4}(\zeta)= \pm \frac{\lambda}{2} \pm \frac{2 \mu}{\sqrt{4 \mu-\lambda^{2}} \cot \left(\frac{1}{2} \sqrt{4 \mu-\lambda^{2}}(\zeta+k)\right)-\lambda}, \\
& v_{4}(\zeta)=\frac{1}{2}\left( \pm \frac{\lambda}{2} \pm \frac{2 \mu}{\sqrt{4 \mu-\lambda^{2}} \cot \left(\frac{1}{2} \sqrt{4 \mu-\lambda^{2}}(\zeta+k)\right)-\lambda}\right)^{2}
\end{aligned}
$$

where

$$
\zeta=x-\left(-\frac{\lambda^{2}}{8}+\frac{\mu}{2}\right) t \text {. }
$$

3) When $\lambda^{2}-4 \mu>0, \mu=0, \lambda \neq 0$;

$$
\begin{gathered}
u_{5}(\zeta)= \pm \frac{\lambda(\exp (\lambda(\zeta+k))+1)}{2(\exp (\lambda(\zeta+k))-1)}, \\
v_{5}(\zeta)=\frac{1}{2}\left( \pm \frac{\lambda(\exp (\lambda(\zeta+k))+1)}{2(\exp (\lambda(\zeta+k))-1)}\right)^{2}
\end{gathered}
$$

where

$$
\zeta=x-\left(-\frac{\lambda^{2}}{8}+\frac{\mu}{2}\right) t .
$$

4) When $\lambda^{2}-4 \mu=0, \mu \neq 0, \lambda \neq 0$;

$$
\begin{gathered}
u_{6}(\zeta)= \pm \frac{\lambda}{\lambda(\zeta+k)+2}, \\
v_{6}(\zeta)=\frac{1}{2}\left( \pm \frac{\lambda}{\lambda(\zeta+k)+2}\right)^{2}
\end{gathered}
$$

where

$$
\zeta=x-\left(-\frac{\lambda^{2}}{8}+\frac{\mu}{2}\right) t \text {. }
$$

5) When $\lambda^{2}-4 \mu=0, \mu=0, \lambda=0$;

$$
\begin{gathered}
u_{7}(\zeta)= \pm \frac{1}{\zeta+k}, \\
v_{7}(\zeta)=\frac{1}{2}\left( \pm \frac{1}{\zeta+k}\right)^{2}
\end{gathered}
$$

where

$$
\zeta=x-\left(-\frac{\lambda^{2}}{8}+\frac{\mu}{2}\right) t .
$$

\subsection{The modified KdV-Zakharov-Kuznetsev equation}

In this subsection we will deal with the $(3+1)$ dimensional modified KdV-Zakharov-Kuznetsev equation's exact solutions. Think that the $(3+1)$ dimensional modified KdV-Zakharov-Kuznetsev equation in the following form

$$
u_{t}+\alpha u^{2} u_{x}+u_{x x x}+u_{x y y}+u_{x z z}=0
$$

(Islam et. al., 2014). If we apply following transformation

$$
u(x, y, t)=u(\zeta), \zeta=x+y-w t,
$$

to Eq. (21) and integrating founded ODE once with respect to $\zeta$, we obtain following ODE

$$
3 u "+\frac{1}{3} \alpha u^{3}-w u=0
$$

where the prime denotes the derivation with respect to $\zeta$ then setting constant of integration to zero. It is seen that homogeneous balance $n=1$ for ODE (22) so we can search exact solution of Eq. (22) in the following form

$$
u(\zeta)=\alpha_{0}+\alpha_{1} \exp (-\phi(\zeta))
$$


If we substitute solution (23) into Eq. (22), then equating the coefficients of $\exp (-\phi(\zeta))^{j}$ to zero $(j \geq 0)$, we obtain

$$
\begin{aligned}
-w \alpha_{0}+\frac{\alpha \alpha_{0}^{3}}{3}+3 \alpha_{1} \mu \lambda & =0 \\
-w \alpha_{1}+\alpha \alpha_{0}^{2} \alpha_{1}+6 \alpha_{1} \mu+3 \alpha_{1} \lambda^{2} & =0 \\
\alpha \alpha_{0} \alpha_{1}^{2}+9 \alpha_{1} \lambda & =0 \\
\frac{\alpha \alpha_{1}^{3}}{3}+6 \alpha_{1} & =0 .
\end{aligned}
$$

When Sys. (24) is solved, we get

$$
\begin{array}{rrr}
w=w, & \alpha_{0}=0, & \alpha_{1}=0, \\
w=\frac{\alpha \alpha_{0}^{2}}{3}, & \alpha_{0}=\alpha_{0}, & \alpha_{1}=0, \\
w=6 \mu-\frac{3 \lambda^{2}}{2}, & \alpha_{0}=6 \sqrt{-\frac{1}{2 \alpha}}, & \alpha_{1}=3 \lambda \sqrt{-\frac{1}{2 \alpha},} \\
w=6 \mu-\frac{3 \lambda^{2}}{2}, & \alpha_{0}=-6 \sqrt{-\frac{1}{2 \alpha}}, & \alpha_{1}=-3 \lambda \sqrt{-\frac{1}{2 \alpha} .} .
\end{array}
$$

We should use only third and fourth cases in (25) because of $\alpha_{1} \neq 0$.

When

$$
w=6 \mu-\frac{3 \lambda^{2}}{2}, \alpha_{0}= \pm 6 \sqrt{-\frac{1}{2 \alpha}}, \alpha_{1}= \pm 3 \lambda \sqrt{-\frac{1}{2 \alpha}},
$$

solution can be given in the following form

$$
u(\zeta)= \pm 6 \sqrt{-\frac{1}{2 \alpha}} \pm 3 \lambda \sqrt{-\frac{1}{2 \alpha}} \exp (-\phi(\zeta))
$$

where

$$
\zeta=x-\left(6 \mu-\frac{3 \lambda^{2}}{2}\right) t .
$$

Then we obtain the following seven exact solutions of Eq. (21).

1) When $\lambda^{2}-4 \mu>0, \mu \neq 0$;

$$
u_{1}(\zeta)= \pm 3 \lambda \sqrt{-\frac{1}{2 \alpha}} \mp \frac{12 \mu \sqrt{-\frac{1}{2 \alpha}}}{\sqrt{\lambda^{2}-4 \mu} \tanh \left(\frac{1}{2} \sqrt{\lambda^{2}-4 \mu}(\zeta+k)\right)+\lambda}
$$

and

$$
u_{2}(\zeta)= \pm 3 \lambda \sqrt{-\frac{1}{2 \alpha}} \mp \frac{12 \mu \sqrt{-\frac{1}{2 \alpha}}}{\sqrt{\lambda^{2}-4 \mu} \operatorname{coth}\left(\frac{1}{2} \sqrt{\lambda^{2}-4 \mu}(\zeta+k)\right)+\lambda}
$$

where $\zeta=x-\left(6 \mu-\frac{3 \lambda^{2}}{2}\right) t$

2) When $\lambda^{2}-4 \mu<0, \mu \neq 0$;

$u_{3}(\zeta)= \pm 3 \lambda \sqrt{-\frac{1}{2 \alpha}} \pm \frac{12 \mu \sqrt{-\frac{1}{2 \alpha}}}{\sqrt{4 \mu-\lambda^{2}} \tan \left(\frac{1}{2} \sqrt{4 \mu-\lambda^{2}}(\zeta+k)\right)-\lambda}$

and

$u_{4}(\zeta)= \pm 3 \lambda \sqrt{-\frac{1}{2 \alpha}} \pm \frac{12 \mu \sqrt{-\frac{1}{2 \alpha}}}{\sqrt{4 \mu-\lambda^{2}} \cot \left(\frac{1}{2} \sqrt{4 \mu-\lambda^{2}}(\zeta+k)\right)-\lambda}$

where

$\zeta=x-\left(6 \mu-\frac{3 \lambda^{2}}{2}\right) t$.

3) When $\lambda^{2}-4 \mu>0, \mu=0, \lambda \neq 0$;

$$
u_{5}(\zeta)= \pm \frac{3}{\sqrt{2}} \frac{\sqrt{-\frac{1}{\alpha}} \lambda(\exp (\lambda(\zeta+k))+1)}{(\exp (\lambda(\zeta+k))-1)}
$$

where

$$
\zeta=x-\left(6 \mu-\frac{3 \lambda^{2}}{2}\right) t .
$$

4) When $\lambda^{2}-4 \mu=0, \mu \neq 0, \lambda \neq 0$,

where

$$
u_{6}(\zeta)= \pm \frac{3 \sqrt{2} \lambda \sqrt{-\frac{1}{\alpha}}}{\lambda(\zeta+k)+2}
$$

$\zeta=x-\left(6 \mu-\frac{3 \lambda^{2}}{2}\right) t$.

5) When $\lambda^{2}-4 \mu=0, \mu=0, \lambda=0$,

$$
u_{7}(\zeta)= \pm \frac{6 \sqrt{-\frac{1}{2 \alpha}}}{\zeta+k}
$$

where

$$
\zeta=x-\left(6 \mu-\frac{3 \lambda^{2}}{2}\right) t \text {. }
$$

\section{Conclusion}

In this paper, we used the $\exp (-\phi(\zeta))$ expansion method for obtaining new exact solutions of the Bogoyavlenskii equation and the modified KdV-Zakharov-Kuznetsev equation. After this application we obtained seven exact solutions for each equation. Solutions are obtained with 
hyperbolic, trigonometric, rational solutions. We checked the correctness of the obtained solutions. Our results show that the method is reliable and effective. Also, it has been seen that our obtained solutions include other obtained solutions in literature. Obtained solutions can be useful for applications in mathematical physics and engineering. Thus, we conclude that method can be useful for solving the different nonlinear partial differential equations and nonlinear fractional order differential equations.

\section{References}

Liu, G.T, Fan, TY., 2005. New applications of developed Jacobi elliptic function expansion methods, Physics Letters A, 345, 161-166.

Achab, AE., 2016. Constructing of exact solutions to the nonlinear Schrödinger equation (NLSE) with power-law nonlinearity by the Weierstrass elliptic function method. Optik, $127,1229-1232$

Matveev, VB., Salle, MA., 1991. Darboux Transformation and Solitons. Berlin: Springer.

Tascan, F., Yakut, A., 2015. Conservation Laws and Exact Solutions with Symmetry Reduction of Nonlinear Reaction Diffusion Equations. International Journal of Nonlinear Sciences and Numerical Simulation, 16, 191-196.

Bluman, GW., Kumei, S.,1989. Symmetries and differential equations. New York, NY, USA: Springer Verlag.

Bekir, A., Cevikel, AC., 2009. Solitary wave solutions of two nonlinear physical models by tanh--coth method. Communications in Nonlinear Science and Numerical Simulation, 14, 1804-1809.

Kaplan, $M$,, Akbulut, A, Bekir, A.,2015. Exact Travelling Wave Solutions of the Nonlinear Evolution Equations by Auxiliary Equation Method. Zeitschrift für Naturforschung A,70, 969-974.

Wazwaz, AM., 2008. The extended tanh method for the Zakharov-Kuznetsov (ZK) equation, the modified ZK equation, and its generalized forms, Communications in Nonlinear Science and Numerical Simulation, 13, 1039-1047.

Taşcan, F., Bekir, A., 2009. Analytic solutions of the $(2+1)$-dimensional nonlinear evolution equations using the sine-cosine method, Applied Mathematics and Computation, 215, 3134-3139.

Eslami, M., Vajargah, BF., Mirzazadeh, M., 2014. Exact solutions of modified Zakharov-Kuznetsov equation by the homogeneous balance method. Ain Shams Engineering Journal ,5, 221-225.

Ghaneai, H., Hosseini. MM.,2016. Solving differential-algebraic equations through variational iteration method with an auxiliary parameter. Applied Mathematical Modelling, 40, 3991-4001.

Zayed, EME.,, Abdelaziz, MAM., 2011. Exact solutions for the nonlinear Schrödinger equation with variable coefficients using the generalized extended tanh-function, the sine-cosine and the exp-function methods, Applied Mathematics and Computation, 218, 22592268.

Khan, K., Akbar, MA., 2014. Traveling wave solutions of nonlinear evolution equations via the enhanced $\left(G^{\prime} / G\right.$ )-expansion method, Journal of the Egyptian Mathematical Society, $22,220-226$.

Kaplan, M., Bekir, A., Akbulut, A., Aksoy, E.,2015. The modified simple equation method for nonlinear fractional differential equations. Romanian Journal of Physics ,60, 1374-1383.

Taghizadeh, N., Mirzazadeh, M., Farahrooz, F., 2011. Exact solutions of the nonlinear Schrödinger equation by the first integral method, Journal of Mathematical Analysis and Applications, 374, 549-553.

Zhang,Z., 2008. New Exact Traveling Wave Solutions for the Nonlinear Klein-Gordon Equation, Turkish Journal of Physics, 32, 235 240.

Khan, K., Akbar, MA., 2014. The $\exp (-\phi(\xi))-$ expansion method for finding travelling wave solutions of Vakhnenko-Parkes equation, 
International Journal of Dynamical Systems and Differential Equations, 5, 72-83.

Khan, K., Akbar, MA., 2013. Application of $\exp (-\phi(\xi))$ - expansion method to find the exact solutions of modified Benjamin-BonaMahony equation. World Applied Sciences Journal, 24, 1373-1377.

Hafez, MG., Ali, MY., Akter, MT., Kauser, MA., 2014. Application of the $\exp (-\phi(\xi))$ expansion method for finding exact solutions of the (1+1)-dimensional dispersive long wave equations. British Journal of Mathematics \& Computer Science , 22, 3191-3201.

Abdelrahman, MAE., Zahran, HM., Khater, MA., 2015. The $\exp (-\phi(\xi))$-expansion method and its application for solving nonlinear evolution equations. International Journal of Modern Nonlinear Theory and Application, 4, 37-47.

Roshid, H.O., Alam, M.N., Akbar M.A., 2015. Traveling Wave Solutions for Fifth Order (1+1)Dimensional Kaup-Kupershmidt Equation with the Help of $\exp (-\phi(\xi))$ Expansion Method. Walailak Journal, 12, 1063-1073.

Khater, M.A., 2016. Exact traveling wave solutions for the generalized Hirota-Satsuma couple KdV system using the $\exp (-\phi(\xi))$ expansion method. Cogent Mathematics, 3, 1172397.

Zahran, E.H.M., Khater, M.A., 2015. Exact Traveling Wave Solutions For Some Nonlinear Evolution Equations by Using the $\exp (-\phi(\xi))$ Expansion Method. Asian Journal of Mathematics and Computer Research, 4, 195207.

Malik, A., Kumar, H., Chand, F., Singh, S., Mischra, S.C.,2010. Exact Traveling Wave Solutions of the Bogoyavlenskii Equation. International Archive of Applied Sciences and Technology, 1, 93-98.

Najafi, M., Arbabi, S., Najafi, M., 2012. New Exact Solutions of $(2+1)$-Dimensional Bogoyavlenskii Equation by the Sine-Cosine Method. International Journal of Basic and Applied Sciences, 1, 490-497.
Naher, H., Abdullah, F.A., Akbar, M.A., 2013. Generalized and Improved (G'/G)-Expansion Method for (3+1)-Dimensional Modified KdVZakharov-Kuznetsev Equation. Plos One, 8, 7 pp.

Hafez, MG., Akbar, MA., 2015. New exact traveling wave solutions to the (1+1)-dimensional KleinGordon-Zakharov equation for wave propagation in plasma using the exp $\exp (-\phi(\xi))$-expansion method. Propulsion and Power Research, 4, 31-39.

Malik, A., Chand, F., Kumar, H., Mishra, SC., 2012. Exact solutions of the Bogoyavlenskii equation using the multiple ( $\mathrm{G}^{\prime} / \mathrm{G}$ )-expansion method, Computers and Mathematics with Applications, 64 : 2850-2859.

Peng, Y., Shen, M., 2006. On exact solutions of the Bogoyavlenskii equation, Pramana-journal of physics , $67: 449-456$.

Islam, Md H., Khan, K., Akbar, MA., and Salam, Md A., 2014. Exact traveling wave solutions of modified KdV-Zakharov-Kuznetsov equation and viscous Burgers equation. SpringerPlus, 3 , 105, $9 \mathrm{pp}$. 